\title{
On the Renormalizability of Theories with Gauge Anomalies
}

\author{
Rodolfo Casana ${ }^{1}$, Sebastião A. Dias ${ }^{1,2}$ \\ ${ }^{1}$ Centro Brasileiro de Pesquisas Físicas \\ Departamento de Campos e Partículas \\ Rua Xavier Sigaud, 150, 22290-180, Rio de Janeiro, Brazil \\ ${ }^{2}$ Pontifícia Universidade Católica do Rio de Janeiro \\ Departamento de Física \\ Rua Marquês de São Vicente, 225, 22543-900, Rio de Janeiro, Brazil
}

\begin{abstract}
We consider the detailed renormalization of two $(1+1)$-dimensional gauge theories which are quantized without preserving gauge invariance: the chiral and the "anomalous" Schwinger models. By regularizing the non-perturbative divergences that appear in fermionic Green's functions of both models, we show that the "tree level" photon propagator is illdefined, thus forcing one to use the complete photon propagator in the loop expansion of these functions. We perform the renormalization of these divergences in both models to one loop level, defining it in a consistent and semi-perturbative sense that we propose in this paper.
\end{abstract}

\section{Introduction}

Cancellation of gauge anomalies is almost a dogma nowadays, when one speaks about consistently defining a quantum gauge theory [1]. Traditional perturbation methods give no hope of defining a finite and consistent theory in the presence of a gauge anomaly, mainly because of the breakdown of Ward (or Slavnov-Taylor) identities, which are extensively used to relate counterterms and cancel divergent diagrams. It became a guiding principle both for experimentalists (remember the search for the top quark [2]) and for theorists (for example, in eliminating concurrent string theories [3]).

In two dimensional space-time, however, indications appeared that the situation could be more subtle. When we consider Weyl fermions interacting minimally with an Abelian gauge field (the chiral Schwinger model (CSM) [4]), a 
consistent and unitary quantum theory emerges in spite of the fact that gauge invariance is lost). The resulting quantized model is dependent on a parameter $a$ (Jackiw-Rajaraman parameter) which is introduced at quantum level, and can not be fixed a priori to any value. It is related to regularization ambiguities of the theory, that can not be removed precisely because there is no "natural" regularization scheme (one that would preserve quantum gauge symmetry).

A similar fact can occur within the context of the traditional Schwinger model (Dirac fermions interacting minimally with an Abelian gauge field [5]). There exist regularizations that preserve gauge invariance in all intermediate steps, and the model, studied this way, provides very rich predictions about several phenomena in Quantum Field Theory [6]-15]. However, if one chooses a regularization that does not preserve explicitly gauge invariance from the beginning [16], one ends with an effective action that is not gauge invariant, but depends on a similar parameter $a$. When one sets $a=1$, one brings back the desired gauge invariance. This is possible because there is no true gauge anomaly in this case (gauge invariance can be restored by the addiction of BRST co-homologically trivial counterterms). In spite of that, even for values of $a$ different from one, the theory is consistent and unitary, thus following closely what happens in the CSM. This model, viewed in this context, is usually called "non-confining" Schwinger model [17]. As the property of confinement depends crucially on the renormalization of the model, thus not being firmly established up to now, we prefer to call it "anomalous" Schwinger model (ASM), just to remember that gauge invariance is not explicitly preserved by regularization.

In addiction to these facts, it has been discovered [18, 19, 20] that there is a kind of symmetry restoration mechanism acting in the background of anomalous gauge theories. This symmetry restoration is possible thanks to the spontaneous appearance of a new set of quantum degrees of freedom, the Wess-Zumino fields, which, after being integrated along with the fermions, help to build a gauge invariant effective action. This can be explicitly demonstrated in $(1+1)$ dimensions, for abelian gauge theories, because the integration over the fermions furnishes a quadratic integrated polynomial in $A_{\mu}$ [20]. For higher dimensions or non-abelian theories this important question remains opened, either because of the complexity of the integration over the fermions [21] or of the quite complicated interactions of the Wess-Zumino fields 22].

This scenario suggests that there is still a lot to be learned about anomalous gauge theories, before discarding them definitely (at least from a theoretical point of view). In fact, even in the $(1+1)$-dimensional case there are unanswered questions. Both in CSM and in ASM it is known that the fermionic Green's functions are divergent (for any value of $a$, in the CSM [23], and for $a \neq 1$ in the ASM [17]). It is readily seen that a fermionic wave function renormalization is sufficient to turn both theories finite [24, 25]. Their renormalization, however, is quite involved, and has never been performed in detail. The main obstacle is that bosonization (necessary for solving the models) is done in posi- 
tion space. It furnishes expressions quite complicated to be Fourier transformed directly (remember that renormalization is usually effected in momenta space). Also, although the divergences are easily seen, they are not so easily regularized, as their origin is not sufficiently clear.

It is our aim, in this paper, to solve these questions and explicitly renormalize both models. We will show that it is possible to do this considering an expansion of the fermion propagator in terms of the full photon propagator (what we call a "semi-perturbative" approach). This has to be preceded by a careful analysis of the structure of the divergences and by regularization, that we also do. We will end with well defined 1PI functions to one loop (in the semi-perturbative expansion).

The paper is organized as follows: in section 2 we present a calculation of the most relevant Green's functions and we show the structure of the divergences. In section 3 we give a regularization for them, in the gauge non-invariant formalism. In section 4 we study the Ward identities and, in section 5 , we perform the renormalization of the model. Finally, in section 6 , we present our conclusions.

\section{Structure of Divergences}

The Schwinger model is defined by the following Lagrangian density $\square$

$$
\mathcal{L}_{V}[\psi, \bar{\psi}, A]=-\frac{1}{4} F_{\mu \nu} F^{\mu \nu}+\bar{\psi}(i \not \partial+e \not A) \psi
$$

The chiral Schwinger model is defined by

$$
\mathcal{L}_{C}[\psi, \bar{\psi}, A]=-\frac{1}{4} F_{\mu \nu} F^{\mu \nu}+\bar{\psi}\left(i \not \partial+e \not A P_{+}\right) \psi
$$

In both models, $\psi$ denotes a two dimensional Dirac fermion. The effective action $W\left[A_{\mu}\right]$ is defined as

$$
e^{i W\left[A_{\mu}\right]}=\int d \psi d \bar{\psi} \exp \left[i \int d x \bar{\psi} i D\left[A_{\mu}\right] \psi\right]=\operatorname{det} i D\left[A_{\mu}\right]
$$

\footnotetext{
${ }^{1}$ Here $d x$ means $d^{2} x$. Our conventions are

$$
\begin{gathered}
g_{\mu \nu}=\left(\begin{array}{cc}
1 & 0 \\
0 & -1
\end{array}\right)=g^{\mu \nu} \quad, \quad \epsilon^{\mu \nu}=\left(\begin{array}{cc}
0 & 1 \\
-1 & 0
\end{array}\right)=-\epsilon_{\mu \nu} . \\
\left\{\gamma^{\mu}, \gamma^{\nu}\right\}=2 g^{\mu \nu}, \gamma_{0}^{\dagger}=\gamma_{0} \quad, \quad \gamma_{1}^{\dagger}=-\gamma_{1} . \\
\left\{\gamma_{\mu}, \gamma_{5}\right\}=0 \quad, \quad \gamma_{5}=\gamma_{0} \gamma_{1} \quad, \quad \gamma_{5}^{\dagger}=\gamma_{5} . \\
P_{ \pm}=\left(1 \pm \gamma_{5}\right) / 2 \quad, \quad \tilde{\partial}_{\mu}=\epsilon_{\mu \nu} \partial^{\nu}
\end{gathered}
$$
}


where

$$
i D\left[A_{\mu}\right]= \begin{cases}i \not \partial+e \not A & , \text { Schwinger model }, \\ i \not \partial+e \not A P_{+} & , \text {chiral Schwinger model . }\end{cases}
$$

We will calculate the fermionic determinant using a prescription which is not gauge invariant [6, 26] for both models. This prescription will be responsible for a value of $a$ different from 1 in the vector case. We known that in the chiral case, there is no gauge invariant prescription, whatever may be the value of $a$.

The effective action in the vectorial case, $W_{V}\left[A_{\mu}\right]$, is given by

$$
W_{V}\left[A_{\mu}\right]=\int d x \frac{1}{2} A_{\mu}(x)\left[m_{V}^{2} g^{\mu \nu}-\frac{e^{2}}{\pi} \frac{\partial^{\mu} \partial^{\nu}}{\square}\right] A_{\nu}(x),
$$

where $m_{V}^{2}$ is the mass dynamically generated for the gauge field $A_{\mu}$,

$$
m_{V}^{2}=\frac{e^{2}}{2 \pi}\left(a_{V}+1\right)
$$

In the chiral case we get the effective action $W_{C}\left[A_{\mu}\right]$, which is

$$
W_{C}[A]=\frac{e^{2}}{8 \pi} \int d x A_{\mu}\left[a_{C} g^{\mu \nu}-\left(\partial_{x}^{\mu}+\tilde{\partial}_{x}^{\mu}\right) \frac{1}{\square}\left(\partial_{x}^{\nu}+\tilde{\partial}_{x}^{\nu}\right)\right] A_{\nu}(x) .
$$

In both cases the parameters $a_{V}$ and $a_{C}$ appear as a consequence of ambiguities in the short-distance regularization of the fermionic determinant.

The generating functional is

$$
Z[\eta, \bar{\eta}, J]=N \int d A_{\mu} d \psi d \bar{\psi} \exp \left[i \int d x\left(\mathcal{L}[\psi, \bar{\psi}, A]+\bar{\eta} \psi+\bar{\psi} \eta+J_{\mu} A^{\mu}\right)\right] .
$$

Now, we can integrate over the fermion fields and get

$$
\begin{aligned}
Z[\eta, \bar{\eta}, J]=\int d A_{\mu} & \exp \left[i \int d x\left(\frac{1}{2} A_{\mu} \Gamma^{\mu \nu} A_{\nu}+J_{\mu} A^{\mu}\right)\right] \\
& \times \exp \left[-i \int d x d y \bar{\eta}(x) G(x, y ; A) \eta(y)\right],
\end{aligned}
$$

where $\Gamma^{\mu \nu}$ is the 1PI two-point function of the gauge field. For the vectorial coupling we have

$$
\Gamma_{V}^{\mu \nu}=g^{\mu \nu}\left(\square+m_{V}^{2}\right)-\partial^{\mu} \partial^{\nu}-\frac{e^{2}}{\pi} \frac{\partial^{\mu} \partial^{\nu}}{\square},
$$

while, in the chiral case,

$$
\Gamma_{C}^{\mu \nu}=g^{\mu \nu}\left(\square+\frac{a_{C} e^{2}}{4 \pi}\right)-\partial^{\mu} \partial^{\nu}-\frac{e^{2}}{4 \pi}\left(\partial^{\mu}+\tilde{\partial}^{\mu}\right) \frac{1}{\square}\left(\partial^{\nu}+\tilde{\partial}^{\nu}\right)
$$


The function $G(x, y ; A)$ in (9), is the two-point fermion Green's function in the external field $A_{\mu}, \quad D\left[A_{\mu}\right] G(x, y ; A)=\delta(x-y)$. This Green's function can be exactly computed in both models:

$$
\begin{array}{r}
G_{V}(x, y ; A)=\exp \left[-i e \int d z A_{\mu}(z) j_{-}^{\mu}(z, x, y)\right] P_{-} G_{F}(x-y)+ \\
+\exp \left[-i e \int d z A_{\mu}(z) j_{+}^{\mu}(z, x, y)\right] P_{+} G_{F}(x-y) . \\
G_{C}(x, y ; A)=\exp \left[-i e \int d z A_{\mu}(z) j_{+}^{\mu}(z, x, y)\right] P_{+} G_{F}(x-y)+P_{-} G_{F}(x-y)
\end{array}
$$

Here, $G_{F}$ satisfies $i \not \partial_{x} G_{F}(x-y)=\delta(x-y)$ and $j_{ \pm}^{\mu}$ is given by

$$
j_{ \pm}^{\mu}(z, x, y)=\left(\partial_{z}^{\mu} \pm \tilde{\partial}_{z}^{\mu}\right)\left[D_{F}(z-x)-D_{F}(z-y)\right],
$$

where $D_{F}(x)$, satisfies $\square D_{F}(x-y)=\delta(x-y)$.

Knowing this, we are able to compute the full photon and fermion propagators. Besides, all the correlation functions of the theory could, in principle, be exactly calculated in configuration space, but not in momentum space, where one does not know how to bosonize directly the theory.

The photon propagator $G_{\mu \nu}(x-y)=\left\langle 0\left|T A_{\mu}(x) A_{\nu}(y)\right| 0\right\rangle$ is straightforwardly computed from (9). When we consider the vectorial coupling, it yields (in momentum space)

$$
i \tilde{G}_{\mu \nu}^{V}(k)=\frac{1}{k^{2}-m_{V}^{2}}\left(g_{\mu \nu}-\frac{k_{\mu} k_{\nu}}{k^{2}}\right)-\frac{2 \pi}{e^{2}\left(a_{V}-1\right)} \frac{k_{\mu} k_{\nu}}{k^{2}},
$$

while, in the chiral case

$$
i \tilde{G}_{\mu \nu}^{C}(k)=\left[g_{\mu \nu}-\frac{k_{\mu} k_{\nu}}{a_{C}-1}\left(\frac{4 \pi}{e^{2}}-\frac{2}{k^{2}}\right)+\frac{k_{\mu} \tilde{k}_{\nu}+\tilde{k}_{\mu} k_{\nu}}{\left(a_{C}-1\right) k^{2}}\right] \frac{1}{k^{2}-m_{C}^{2}},
$$

where $m_{C}^{2}$ is defined as

$$
m_{C}^{2}=\frac{e^{2} a_{C}^{2}}{4 \pi\left(a_{C}-1\right)} .
$$

We observe that this propagator has a pole in $m_{V}^{2}\left(m_{C}^{2}\right)$, that is, the photon acquires mass after the quantization of the theory. We observe the explicit dependence of the photon mass on $a_{V}\left(a_{C}\right)$, which leaves it indefinite. Moreover, the photon propagator is divergence free. Its high-momentum behavior is similar to the one in Proca's theory.

Now we calculate the fermion propagator, $G(x-y)=\langle 0|T \psi(x) \bar{\psi}(y)| 0\rangle$. The vectorial fermion propagator is

$$
\begin{aligned}
G_{V}(x-y)= & i \exp \left\{-\frac{4 \pi i}{a_{V}^{2}-1} \int \frac{d k}{(2 \pi)^{2}} \frac{1-e^{-i k \cdot(x-y)}}{k^{2}}\right\} \\
& \times \exp \left\{-\frac{2 \pi i}{a_{V}+1} \int \frac{d k}{(2 \pi)^{2}} \frac{1-e^{-i k \cdot(x-y)}}{k^{2}-m_{V}^{2}}\right\} G_{F}(x-y),
\end{aligned}
$$


and the chiral fermion propagator is found to be

$$
\begin{aligned}
G_{C}(x-y)=i & \exp \left\{-\frac{4 \pi i}{a_{C}-1} \int \frac{d k}{(2 \pi)^{2}} \frac{1-e^{-i k \cdot(x-y)}}{k^{2}-m_{C}^{2}}\right\} P_{+} G_{F}(x-y)+ \\
+ & i P_{-} G_{F}(x-y) .
\end{aligned}
$$

From (18) and (19), we see that the fermionic propagators have an UV logarithmic divergence, but are free of IR divergences.

This divergence is better understood in momentum space. Although we are not able to compute the Fourier transform exactly, we can still write down the Schwinger-Dyson equation satisfied in the vectorial case,

$$
\left(\not \partial_{x}+e^{2} \int \frac{d k}{(2 \pi)^{2}} f_{V}(k) \not k e^{-i k \cdot(x-y)}\right) G_{V}(x-y)=\delta(x-y),
$$

where $f_{V}(k)$ is given by

$$
\begin{aligned}
f_{V}(k) & =-\frac{4 \pi}{e^{2}\left(a_{V}^{2}-1\right)} \frac{1}{k^{2}}-\frac{2 \pi}{e^{2}\left(a_{V}+1\right)} \frac{1}{k^{2}-m_{V}^{2}} \\
& =-\frac{2 \pi}{e^{2}\left(a_{V}-1\right)} \frac{1}{k^{2}}-\frac{1}{k^{2}\left(k^{2}-m_{V}^{2}\right)} .
\end{aligned}
$$

The Schwinger-Dyson equations for the chiral case have to be written separately for the right and left-handed fermions, as they have different propagators. The right-handed part, $P_{+} G_{C}=G_{C}^{+}$, satisfies

$$
\left(\not \partial_{x}+e^{2} \int \frac{d k}{(2 \pi)^{2}} f_{C}(k) \not k e^{-i k \cdot(x-y)}\right) G_{C}^{+}(x-y)=P_{-} \delta(x-y),
$$

and, for the left-handed part, $P_{-} G_{C}=G_{C}^{-}$, we obtain

$$
\not_{x} G_{C}^{-}(x-y)=P_{+} \delta(x-y) .
$$

It denotes a free left-handed fermion. The function $f_{C}$ is given by

$$
f_{C}(k)=-\frac{4 \pi}{e^{2}\left(a_{C}-1\right)} \frac{1}{k^{2}-m_{C}^{2}} .
$$

We can express both equations, (20) and (23), in a compact way

$$
\left(\not \partial_{x}+e^{2} \int \frac{d k}{(2 \pi)^{2}} f(k) \not k e^{-i k \cdot(x-y)}\right) G(x-y)=\delta(x-y)
$$

This equation can be easily written in momentum space, where it allows one to find a recursive equation for the fermion propagator $\tilde{G}(p)$

$$
\tilde{G}(p)=\frac{i}{\not p}-i e^{2} \int \frac{d k}{(2 \pi)^{2}} f(k) \frac{1}{\not p} \not k \tilde{G}(p-k) .
$$


The consequences of the previous equation will be studied in great detail in one of the next sections. Now, we move to the three-point Green's function, $G^{\mu}(x, y, z)=\left\langle 0\left|T \psi(x) \bar{\psi}(y) A^{\mu}(z)\right| 0\right\rangle$. In the vectorial case, we get

$$
G_{V}^{\mu}(x, y, z)=i e \int \frac{d k}{(2 \pi)^{2}} g_{V}^{\mu}(k)\left[e^{-i k \cdot(z-x)}-e^{-i k \cdot(z-y)}\right] G_{V}(x-y),
$$

with $g_{V}^{\mu}$ given by

$$
g_{V}^{\mu}(k)=\frac{2 \pi k^{\mu}}{e^{2}\left(a_{V}-1\right) k^{2}}-\frac{\gamma_{5} \tilde{k}^{\mu}}{k^{2}\left(k^{2}-m_{V}^{2}\right)} .
$$

In momentum space $\tilde{G}_{V}^{\mu}(p,-p-q, q) \equiv \tilde{G}_{V}^{\mu}(p, q)$

$$
\tilde{G}_{V}^{\mu}(p, q)=i e g_{V}^{\mu}(q)\left[\tilde{G}_{V}(p+q)-\tilde{G}_{V}(p)\right] .
$$

The three-point function for the chiral case is

$$
G_{C}^{\mu}(x, y, z)=i e \int \frac{d k}{(2 \pi)^{2}} g_{C}^{\mu}(k)\left[e^{-i k \cdot(z-x)}-e^{-i k \cdot(z-y)}\right] G_{C}^{+}(x-y),
$$

with $g_{C}^{\mu}$ given by

$$
g_{C}^{\mu}(k)=\frac{4 \pi}{e^{2}\left(a_{C}-1\right)} \frac{k^{\mu}}{k^{2}-m_{C}^{2}}-\frac{a_{C}}{a_{C}-1} \frac{k^{\mu}+\tilde{k}^{\mu}}{k^{2}\left(k^{2}-m_{C}^{2}\right)} .
$$

In momentum space $\tilde{G}_{C}^{\mu}(p,-p-q, q) \equiv \tilde{G}_{C}^{\mu}(p, q)$

$$
\tilde{G}_{C}^{\mu}(p, q)=i \operatorname{eg} g_{C}^{\mu}(q)\left[\tilde{G}_{C}^{+}(p+q)-\tilde{G}_{C}^{+}(p)\right] .
$$

We see that the divergence in this function is due to the fermionic propagator. It can be easily seen that only Green's functions with fermionic legs will have UV divergences [23] in both models. A careful analysis leads us to the conclusion that these UV divergences do not have perturbative origin [27 as we are going to see in the next section.

We call attention to the fact that one can write the three-point functions, in both theories, almost entirely in terms of the two-point functions. This is certainly not an accident. Recently Adam 28] and Radozycki and Namyslowski [29] showed, in the context of the conventional (gauge invariantly quantized) Schwinger model, that this property is linked to a reduction of the infinite set of Schwinger-Dyson equations, so that it is possible to express every $n$-point Green's functions in terms of other ones, associated to lower $n$ 's. In their paper, Radozycki and Namyslowski used crucially the fact that gauge invariance was preserved (in the form of a non-anomalous Ward identity for the photon twopoint function). The fact that the same equations are still valid for anomalous models suggest that this fact is not so much dependent on intermediate gauge invariance as could seem at first sight. We will see that this "factorization" is, in fact, a consequence of Ward identities that are preserved also in the anomalous case, and is very important for the renormalizability of the models. 


\section{Regularization}

In this section we will begin by regularizing the vectorial theory. We will use the point of view which is called gauge non-invariant formalism 222 where one does not introduce a Wess-Zumino field to restore gauge symmetry [18, 20. However, at least for the case dealt with in this paper, the results are coincident [27]. In the gauge non-invariant formalism, the vector field $A_{\mu}$ is decomposed in its longitudinal and transverse parts, so

$$
e A_{\mu}=\partial_{\mu} \rho-\tilde{\partial}_{\mu} \phi
$$

If we substitute the decomposition (34) in the action of both models, we notice that, thanks to classical gauge invariance, there is no kinetical term for the field $\rho$, that represents the longitudinal part of $A_{\mu}$. We could try to decouple this field, making a change of fermionic variables,

$$
\psi=e^{i \rho} \psi^{\prime}, \bar{\psi}=\bar{\psi}^{\prime} e^{-i \rho} .
$$

However, the fermionic measure is not invariant under (35), and changes as

$$
d \psi d \bar{\psi}=d \psi^{\prime} d \bar{\psi}^{\prime} \exp \left[\frac{i\left(a_{V}-1\right)}{4 \pi} \int d x \partial_{\mu} \rho \partial^{\mu} \rho\right]
$$

thus giving a kinetical term for the $\rho$ field. Putting into the generating functional (8), we obtain

$$
\begin{aligned}
Z_{V}[\eta, \bar{\eta}, J]= & \int d \rho d \phi d \psi d \bar{\psi} \exp \left[i \int d x \left(\frac{1}{2 e^{2}} \phi \square^{2} \phi+\bar{\psi}(i \not \partial-\tilde{\partial} \phi) \psi+\right.\right. \\
& \left.\left.+\frac{\left(a_{V}-1\right)}{4 \pi} \partial_{\mu} \rho \partial^{\mu} \rho+\bar{\eta} e^{i \rho} \psi+\bar{\psi} e^{-i \rho} \eta+\frac{1}{e} J_{\mu} \partial^{\mu} \rho-\frac{1}{e} J_{\mu} \tilde{\partial}^{\mu} \phi\right)\right] .
\end{aligned}
$$

We notice that our tentative of decoupling was not successful, but had the effect of generating a kinetical term to $\rho$. This field is still coupled to the fermion fields, in a very complicated way, through the fermionic sources. This is not a usually noticed detail, as many people use to work with the vacuum-vacuum amplitude, which has the sources set to zero. This coupling induces the UV divergence. We will show this explicitly for the vector case, the chiral case being completely analogous. To compute the fermion two point function, one has to take two functional derivatives of $Z$, given by equation (37), with respect to $\eta$ and $\bar{\eta}$. Doing this and, in the following, putting the sources to zero we obtain

$$
\begin{aligned}
0|T \psi(x) \bar{\psi}(y)| 0\rangle= & \int d \rho d \phi d \psi d \bar{\psi} \psi(x) \bar{\psi}(y) \exp \left[i \int d x \left(\frac{1}{2 e^{2}} \phi \square^{2} \phi+\right.\right. \\
& \left.\left.+\bar{\psi}(i \not \partial-\tilde{\phi} \phi) \psi+\frac{\left(a_{V}-1\right)}{4 \pi} \partial_{\mu} \rho \partial^{\mu} \rho+i \int d z \rho(z) j(z, x, y)\right)\right],
\end{aligned}
$$


where $j(z, x, y)=\delta(z-x)-\delta(z-y)$. We notice the quadratic dependence of this expression in $\rho$, which enables its exact integration. If one performs it naively, one finds a factor

$$
\exp \left\{-\frac{2 \pi i}{a-1} \int \frac{d k}{(2 \pi)^{2}} \frac{1-e^{-i k \cdot(x-y)}}{k^{2}}\right\}
$$

which comes before the free fermion propagator, that is logarithmically divergent. This divergence has an essentially non-perturbative nature, as can be seen by the perturbative evaluation of the fermion two-point function (there is no individual Feynman diagram inducing this divergence) and has its parallel in the so called gauge invariant formalism, where one can see the same divergence appearing by the exact integration over the Wess-Zumino fields [27].

Having identified the origin of the divergence, the next step is to regularize it. The fundamental observation here, is that we could make everything finite if we had a better UV behavior for the $\rho$ propagator. We can do this by means of Pauli-Villars regularization. We add to the generating functional a new field $\beta$ which has a large mass $\Lambda^{2}$ [30 $\left(\Lambda^{2} \rightarrow+\infty\right)$. This field can be added according to the usual recipe: a) add a term

$$
\frac{\left(a_{V}-1\right)}{4 \pi} \beta\left(\square+\Lambda^{2}\right) \beta
$$

to the action; b) modify the interaction terms of $\rho$ through the substitution $\rho \rightarrow \rho+\beta$; c) functionally integrate over $\beta$ (immediately before this step, one performs the change of variables in the $\rho$ integration $\rho^{\prime}=\rho-\beta$ ). This defines the regularized generating functional $Z_{V}^{\Lambda}[\eta, \bar{\eta}, J]$, which reduces to $Z_{V}[\eta, \bar{\eta}, J]$ in the limit of infinite $\Lambda$. After the indicated manipulations into the regularized generating functional, we get

$$
\begin{array}{r}
Z_{V}^{\Lambda}[\eta, \bar{\eta}, J]=\int d \rho d \phi d \psi d \bar{\psi} \exp \left[i \int d x \left(\frac{1}{2 e^{2}} \phi \square^{2} \phi+\bar{\psi}(i \not \partial-\tilde{\phi} \phi) \psi+\right.\right. \\
\left.\left.-\frac{\left(a_{V}-1\right)}{4 \pi \Lambda^{2}} \rho \square\left(\square+\Lambda^{2}\right) \rho+\bar{\eta} e^{i \rho} \psi+\bar{\psi} e^{-i \rho} \eta+\frac{1}{e} J \cdot \partial \rho-\frac{1}{e} J \cdot \tilde{\partial} \phi\right)\right] .
\end{array}
$$

Now the propagator of the $\rho$ field has a better UV behavior $\left(k^{-4}\right.$, for finite $\left.\Lambda^{2}\right)$. We are interested in the way the original action changes after regularization. Then, we come back to the original fields of the theory,

$$
\psi=e^{-i \rho} \psi^{\prime} \quad, \quad \bar{\psi}=\bar{\psi}^{\prime} e^{i \rho}
$$

(remembering that we have to take into account again the Jacobian) and perform the inverse transformations $\rho=\frac{\partial_{\mu}}{\square} A^{\mu}, \phi=\frac{\tilde{\partial}_{\mu}}{\square} A^{\mu}$, to get the regularized generating functional in terms of the original fields of the theory (1)),

$$
Z_{V}^{\Lambda}[\eta, \bar{\eta}, J]=\int d A_{\mu} d \psi d \bar{\psi} \exp \left(i \int d x \mathcal{L}_{V}^{\Lambda}\left[\psi, \bar{\psi}, A_{\mu}\right]+J \cdot A+\bar{\eta} \psi+\bar{\psi} \eta\right),
$$


where $\mathcal{L}_{V}^{\Lambda}\left[\psi, \bar{\psi}, A_{\mu}\right]$ is the Lagrangian density for the regularized theory

$$
\mathcal{L}_{V}^{\Lambda}\left[\psi, \bar{\psi}, A_{\mu}\right]=-\frac{1}{4} F_{\mu \nu} F^{\mu \nu}+\bar{\psi}(i \not \partial+e \not A) \psi-\frac{e^{2}\left(a_{V}-1\right)}{4 \pi \Lambda^{2}}(\partial \cdot A)^{2}
$$

We see a new term into the Lagrangian density, equivalent to a "gauge fixing" condition with a gauge parameter which is $a$ dependent and proportional to $\Lambda^{-2}$. This new term allows us to regularize the full theory.

Following the same steps we can regularize also the chiral theory. We obtain

$$
\mathcal{L}_{C}^{\Lambda}\left[\psi, \bar{\psi}, A_{\mu}\right]=-\frac{1}{4} F_{\mu \nu} F^{\mu \nu}+\bar{\psi}\left(i \not \partial+e \not A P_{+}\right) \psi-\frac{e^{2}\left(a_{C}-1\right)}{8 \pi \Lambda^{2}}(\partial \cdot A)^{2} .
$$

which also differs from the initial chiral Lagrangian density (2) by a "gauge fixing" term, just like it happened in the vectorial case.

In both cases, we should remember at this point that we do not have the right to fix the gauge, as both theories are anomalous. This is not what is being done. It is, in fact, quite curious that the regularization can be performed in a way that is similar to a gauge fixing.

From (43), we can now calculate the regularized Green's functions. First, we find the Green's functions of the vectorial case. The photon propagator is

$$
i \tilde{G}_{V \mu \nu}^{\Lambda}(k)=\frac{g_{\mu \nu}}{k^{2}-m_{V}^{2}\left(a_{V}\right)}+f_{V}^{\Lambda}(k) k_{\mu} k_{\nu}
$$

where the function $f_{V}^{\Lambda}$ is

$$
f_{V}^{\Lambda}(k)=\frac{2 \pi \Lambda^{2}}{e^{2}\left(a_{V}-1\right)} \frac{1}{k^{2}\left(k^{2}-\Lambda^{2}\right)}-\frac{1}{k^{2}\left(k^{2}-m_{V}^{2}\right)}
$$

which is finite when $\Lambda^{2} \rightarrow+\infty$, as in the non-regularized theory. The high momentum behavior of the regularized photon propagator $\left(k^{-2}\right)$ is better than in the non regularized case. This allows the regularization of the fermion propagator, as we will see.

The regularized fermion propagator is given by

$$
G_{V}^{\Lambda}(x-y)=i \exp \left\{i e^{2} \int \frac{d k}{(2 \pi)^{2}} f_{V}^{\Lambda}(k)\left[1-e^{-i k \cdot(x-y)}\right]\right\} G_{F}(x-y),
$$

and satisfies the Schwinger-Dyson equation (26). In momentum space the regularized fermion propagator $\tilde{G}_{V}^{\Lambda}(p)$ also satisfies equation (27). A power counting analysis of the terms above shows that the UV logarithmic divergence is controlled. The regularized 1PI two-point fermionic function is

$$
\tilde{\Gamma}_{V}^{\Lambda}(p)=\not p\left[1+i \hbar e^{2} \int \frac{d k}{(2 \pi)^{2}} f_{V}^{\Lambda}(k) \not k \frac{1}{p-\not k}+\mathcal{O}\left(\hbar^{2}\right)\right]
$$


and the regularized three-point function

$$
G_{V}^{\Lambda} \mu(x, y, z)=i e \int \frac{d k}{(2 \pi)^{2}} g_{V}^{\Lambda} \mu(k)\left[e^{-i k \cdot(z-x)}-e^{-i k \cdot(z-y)}\right] G_{V}^{\Lambda}(x-y),
$$

with $g_{V}^{\Lambda} \mu$ is given by

$$
g_{V}^{\Lambda} \mu(k)=-\frac{2 \pi \Lambda^{2}}{e^{2}\left(a_{V}-1\right)} \frac{k^{\mu}}{k^{2}\left(k^{2}-\Lambda^{2}\right)}-\frac{\gamma_{5} \tilde{k}^{\mu}}{k^{2}\left(k^{2}-m_{V}^{2}\right)} .
$$

In momentum space $\tilde{G}_{V}^{\Lambda} \mu(p,-p-q, q) \equiv \tilde{G}_{V}^{\Lambda} \mu(p, q)$ satisfies equation (30). And the regularized 1PI three-point function $\tilde{\Gamma}_{V}^{\Lambda} \mu(p, q)$ is

$$
\tilde{\Gamma}_{V}^{\Lambda} \mu(p, q)=e \frac{\gamma^{\mu} \not}{q^{2}}\left[\tilde{\Gamma}_{V}^{\Lambda}(p+q)-\tilde{\Gamma}_{V}^{\Lambda}(p)\right] .
$$

So, we showed that the 1PI three-point function is regularized if the 1PI two-point fermionic function is regularized, as expected. It is easy to show, in a similar way, that all the fermionic Green's functions are regularized too. Thus, we only need to renormalize the fermion two-point function, as we will do in the next sections.

For the chiral case, the regularized Green's functions are also easily computed. The regularized gauge field propagator is

$$
\begin{gathered}
i \tilde{G}_{C \mu \nu}^{\Lambda}(k)=\frac{g_{\mu \nu}\left(k^{2}-\Lambda^{2}\right)}{\left(k^{2}-\omega_{m}\right)\left(k^{2}-\omega_{\Lambda}\right)}-\frac{1}{a_{C}-1} \frac{\Lambda^{2}\left(k_{\mu} \tilde{k}_{\nu}+\tilde{k}_{\mu} k_{\nu}\right)}{k^{2}\left(k^{2}-\omega_{m}\right)\left(k^{2}-\omega_{\Lambda}\right)}+ \\
+\frac{k_{\mu} k_{\nu}}{k^{2}\left(k^{2}-\Lambda^{2}\right)}\left[\frac{4 \pi \Lambda^{2}}{e^{2}\left(a_{C}-1\right)}-\frac{\left(k^{2}-\Lambda^{2}\right)^{2}}{\left(k^{2}-\omega_{m}\right)\left(k^{2}-\omega_{\Lambda}\right)}-\frac{\Lambda^{4} /\left(a_{C}-1\right)^{2}}{\left(k^{2}-\omega_{m}\right)\left(k^{2}-\omega_{\Lambda}\right)}\right],
\end{gathered}
$$

where $\omega_{m}$ and $\omega_{\Lambda}$ satisfy

$$
\begin{aligned}
\omega_{\Lambda}+\omega_{m} & =\Lambda^{2}+\frac{e^{2}\left(a_{C}+1\right)}{4 \pi} \\
\omega_{\Lambda} \omega_{m} & =\Lambda^{2} m_{C}^{2} .
\end{aligned}
$$

We can solve these equations, to obtain

$$
\begin{aligned}
& \omega_{\Lambda} \approx \Lambda^{2}-\frac{e^{2}}{4 \pi\left(a_{C}-1\right)}+\mathcal{O}\left(\Lambda^{-2}\right) \\
& \omega_{m} \approx m_{C}^{2}+\mathcal{O}\left(\Lambda^{-2}\right) .
\end{aligned}
$$

The regularized right-handed fermion propagator is given by

$$
G_{C}^{\Lambda}+(x-y)=i \exp \left(i e^{2} \int \frac{d k}{(2 \pi)^{2}} f_{C}^{\Lambda}(k)\left[1-e^{-i k \cdot(x-y)}\right]\right) P_{+} G_{F}(x-y)
$$


and the left-handed fermion propagator is, $G_{C}^{\Lambda_{-}}(x-y)=i P_{-} G_{F}(x-y)$. The function $f_{C}^{\Lambda}$ is defined as

$$
f_{C}^{\Lambda}(k)=\frac{4 \pi \Lambda^{2}}{e^{2}\left(a_{C}-1\right)} \frac{1}{k^{2}\left(k^{2}-\Lambda^{2}\right)}-\frac{\left[k^{2}-a_{C} \Lambda^{2} /\left(a_{C}-1\right)\right]^{2}}{k^{2}\left(k^{2}-\omega_{m}\right)\left(k^{2}-\omega_{\Lambda}\right)\left(k^{2}-\Lambda^{2}\right)} .
$$

The regularized 3 -point function for the chiral case is

$$
G_{C}^{\Lambda} \mu(x, y, z)=i e \int \frac{d k}{(2 \pi)^{2}} g_{C}^{\Lambda} \mu(k)\left[e^{-i k \cdot(z-x)}-e^{-i k \cdot(z-y)}\right] G_{C}^{\Lambda_{+}}(x-y),
$$

with $g_{C}^{\Lambda} \mu$ given by

$$
g_{C}^{\Lambda} \mu(k)=-k^{\mu} f_{C}^{\Lambda}(k)-\frac{\left(k^{\mu}+\tilde{k}^{\mu}\right)\left[k^{2}-a_{C} \Lambda^{2} /\left(a_{C}-1\right)\right]}{k^{2}\left(k^{2}-\omega_{m}\right)\left(k^{2}-\omega_{\Lambda}\right)} .
$$

In momentum space $\tilde{G}_{C}^{\Lambda} \mu(p,-p-q, q) \equiv \tilde{G}_{C}^{\Lambda} \mu(p, q)$ and we can write

$$
\tilde{G}_{C}^{\Lambda} \mu(p, q)=i \operatorname{eg}_{C}^{\Lambda} \mu(q)\left[\tilde{G}_{C}^{\Lambda}+(p+q)-\tilde{G}_{C}^{\Lambda}+(p)\right],
$$

the regularized 1PI three-point function being

$$
\tilde{\Gamma}_{C}^{\Lambda} \mu(p, q)=e \frac{\left(q^{\mu}+\tilde{q}^{\mu}\right)}{q^{2}}\left[P_{-} \tilde{\Gamma}_{C}^{\Lambda}(p+q)-\tilde{\Gamma}_{C}^{\Lambda}(p) P_{+}\right]
$$

\section{Ward Identities}

In this section, we compute the Ward identities of both theories, using the regularized Lagrangian densities $\mathcal{L}_{V}^{\Lambda}$ (eq. (44)) and $\mathcal{L}_{C}^{\Lambda}$ (eq. (45)). As is usual, we perform the following gauge transformation in the regularized generating functional, for the gauge field

$$
A_{\mu} \rightarrow A_{\mu}+\frac{1}{e} \partial_{\mu} \lambda(x) .
$$

The fermion transformation depends on the model. In the vectorial case, it is given by

$$
\psi \rightarrow \psi+i \lambda(x) \psi, \quad \bar{\psi} \rightarrow \bar{\psi}-i \lambda(x) \bar{\psi}
$$

whereas in the chiral case

$$
\psi \rightarrow \psi+i \lambda(x) P_{+} \psi, \quad \bar{\psi} \rightarrow \bar{\psi}-i \lambda(x) \bar{\psi} P_{-},
$$

with infinitesimal $\lambda(x)$. In our framework, the fermionic measure is not gauge invariant, changing as $d \psi d \bar{\psi} \rightarrow J\left[A_{\mu}\right] d \psi d \bar{\psi}$. J is the Jacobian of the variable transformations (62) or (63). The vectorial jacobian $J_{V}$ is

$$
\ln J_{V}=-\frac{i\left(a_{V}-1\right)}{2 \pi} \int d x e \lambda \partial \cdot A+\mathcal{O}\left(\lambda^{2}\right)
$$


and the chiral jacobian is

$$
\ln J_{C}=-\frac{i}{4 \pi} \int d x e \lambda\left[\left(a_{C}-1\right) \partial \cdot A-\tilde{\partial} \cdot A\right]+\mathcal{O}\left(\lambda^{2}\right) .
$$

Doing this, it is easy to obtain the fundamental Ward identity satisfied by the generating functional of the 1 PI functions $\Gamma^{\Lambda}\left[\psi, \bar{\psi}, A_{\mu}\right]$. The Ward identity for the vectorial case, is

$$
i \frac{\delta \Gamma_{V}^{\Lambda}}{\delta \psi} \psi-i \frac{\delta \Gamma_{V}^{\Lambda}}{\delta \bar{\psi}} \bar{\psi}+\frac{1}{e} \partial^{\mu} \frac{\delta \Gamma_{V}^{\Lambda}}{\delta A^{\mu}}=\frac{e}{2 \pi}\left(a_{V}-1\right)\left(1+\frac{\square}{\Lambda^{2}}\right) \partial^{\mu} A_{\mu}
$$

while, for the chiral case,

$$
i \frac{\delta \Gamma_{C}^{\Lambda}}{\delta \psi} P_{+} \psi-i P_{-} \frac{\delta \Gamma_{C}^{\Lambda}}{\delta \bar{\psi}} \bar{\psi}+\frac{1}{e} \partial^{\mu} \frac{\delta \Gamma_{C}^{\Lambda}}{\delta A^{\mu}}=\frac{e}{4 \pi}\left[\left(a_{C}-1\right)\left(1+\frac{\square}{\Lambda^{2}}\right) \partial^{\mu}-\tilde{\partial}^{\mu}\right] A_{\mu} .
$$

So, the 1PI two-point bosonic function satisfies (in momentum space), for the vectorial case,

$$
k_{\mu} \tilde{\Gamma}_{V}^{\Lambda \mu \nu}(k)=\frac{e^{2}}{2 \pi}\left(a_{V}-1\right)\left(1-\frac{k^{2}}{\Lambda^{2}}\right) k^{\nu}
$$

and for the chiral case

$$
k_{\mu} \tilde{\Gamma}_{C}^{\Lambda} \mu \nu(k)=\frac{e^{2}}{4 \pi}\left[\left(a_{C}-1\right)\left(1-\frac{k^{2}}{\Lambda^{2}}\right) k^{\nu}-\tilde{k}^{\nu}\right] .
$$

We see the non-transversality of the photon propagator, which is the sign of a gauge anomaly. Proceeding, we obtain another important Ward identity, involving the 1PI two-point fermionic function and the 1PI three-point function. For the vector case we get

$$
\frac{1}{e} q_{\mu} \tilde{\Gamma}_{V}^{\Lambda} \mu(p, q)=\tilde{\Gamma}_{V}^{\Lambda}(p+q)-\tilde{\Gamma}_{V}^{\Lambda}(p)
$$

and for the chiral case

$$
\frac{1}{e} q_{\mu} \tilde{\Gamma}_{C}^{\Lambda} \mu(p, q)=P_{-} \tilde{\Gamma}_{C}^{\Lambda}(p+q)-\tilde{\Gamma}_{C}^{\Lambda}(p) P_{+} .
$$

This identity can be obtained by direct manipulation from equations (51) and (60) respectively. It shows that we only need to renormalize the 1PI two-point fermionic function. On the other hand, it repeats the results already found by explicit computation of the three point functions, thus establishing the origin of the previously mentioned result. This Ward identity will be important for the analysis of the renormalizability of both theories. 


\section{Renormalization}

We will concentrate our analysis in the vectorial case, presenting the results for the chiral case only at the end. In the usual way, we express the regularized Lagrangian density (44) in terms of renormalized quantities and their respective renormalization constants

$$
\mathcal{L}_{V}^{\Lambda}=-\frac{1}{4} Z_{A} F_{\mu \nu} F^{\mu \nu}-\frac{Z_{e}^{2}}{Z_{\psi}^{2}} \frac{e^{2}\left(a_{V}-1\right)}{4 \pi \Lambda^{2}}\left(\partial_{\mu} A^{\mu}\right)^{2}+Z_{\psi} \bar{\psi} i \not \partial \psi+Z_{e} e A_{\mu} \bar{\psi} \gamma^{\mu} \psi
$$

We define the bare fields $A_{o}^{\mu}$ and $\psi_{o}$, and the bare coupling constant $e_{o}$ as

$$
A_{o}^{\mu}=\sqrt{Z_{A}} A^{\mu} \quad, \quad \psi_{o}=\sqrt{Z_{\psi}} \psi \quad, \quad e_{o}=\frac{Z_{e}}{Z_{\psi} Z_{A}^{1 / 2}} e .
$$

The object of the renormalization procedure is to determine $Z_{\psi}, Z_{A}$ and $Z_{e}$ that make all Green's functions of the theory finite. Possible ambiguities in the choice of these constants, are parameterized through the imposition of renormalization conditions.

The pure bosonic Green's functions do not have UV divergences. Then, we do not need counter-terms to renormalize them, which means

$$
Z_{A}=1
$$

We remember the Ward identity (70) satisfied by 1PI bare functions

$$
\frac{1}{e_{o}} q_{\mu} \tilde{\Gamma}_{V}^{\Lambda} \mu(p, q)=\tilde{\Gamma}_{V}^{\Lambda}(p+q)-\tilde{\Gamma}_{V}^{\Lambda}(p) .
$$

Substituting in this equation the relation between the bare and renormalized 1PI functions,

$$
\begin{aligned}
& \tilde{\Gamma}_{V}^{R}(p)=Z_{\psi} \tilde{\Gamma}_{V}^{\Lambda}(p) \\
& \tilde{\Gamma}_{V}^{R} \mu(p, q)=Z_{e} \tilde{\Gamma}_{V}^{\Lambda} \mu(p, q) \text {, }
\end{aligned}
$$

we obtain

$$
\frac{1}{e} q_{\mu} \tilde{\Gamma}_{V}^{R} \mu(p, q)=\left(Z_{e} Z_{\psi}^{-1}\right)^{2}\left[\tilde{\Gamma}_{V}^{R}(p+q)-\tilde{\Gamma}_{V}^{R}(p)\right]
$$

On the other side, if we had started directly with the Lagrangian written in terms of renormalized quantities, equation (72), we could verify that the renormalized functions also satisfy the Ward identity

$$
\frac{1}{e} q_{\mu} \tilde{\Gamma}_{V}^{R} \mu(p, q)=Z_{e} Z_{\psi}^{-1}\left[\tilde{\Gamma}_{V}^{R}(p+q)-\tilde{\Gamma}_{V}^{R}(p)\right]
$$


If we compare equations $(\sqrt{78})$ and $(\sqrt{79})$, we obtain

$$
Z_{e}=Z_{\psi}
$$

Coming back to equation $(\overline{73})$, and remembering that $Z_{A}=1$, we see that

$$
e_{o}=e
$$

We see that the coupling constant of the theory is not renormalized, even when the theory is gauge non-invariantly quantized. This shows that the universality of the electromagnetic interaction, usually expressed by $e A^{\mu}=e_{o} A_{o}^{\mu}$, can be preserved into a gauge non-invariant renormalization scheme. In particular, we see that the coupling constant will not depend on the energy scale $\mu$ selected to impose the renormalization conditions. Hence, we have a null Callan-Symanzik beta function

$$
\beta=\mu \frac{\partial}{\partial \mu} e(\mu) \quad \Rightarrow \quad \beta=0
$$

Analogous conclusions are reached also for the chiral case.

\section{$5.1 \quad$ Semi-perturbative analysis}

We start again from the Lagrangian density $\mathcal{L}_{V}^{\Lambda}\left[\psi, \bar{\psi}, A_{\mu}\right]$ (44),

$$
\mathcal{L}_{V}^{\Lambda}\left[\psi, \bar{\psi}, A_{\mu}\right]=-\frac{1}{4} F_{\mu \nu} F^{\mu \nu}-\frac{e^{2}\left(a_{V}-1\right)}{4 \pi \Lambda^{2}}(\partial \cdot A)^{2}+\bar{\psi}(i \not \partial+e \not A) \psi
$$

With a perturbative calculation in mind, we read the free photon propagator

$$
i \tilde{G}_{\mu \nu}^{0}(k)=\frac{g_{\mu \nu}}{k^{2}}+\frac{k_{\mu} k_{\nu}}{k^{4}}\left(\frac{2 \pi \Lambda^{2}}{e^{2}\left(a_{V}-1\right)}-1\right) .
$$

This propagator (84) diverges quadratically in the limit $\Lambda \rightarrow+\infty$. If we insert it in the perturbative calculation of the correlation functions, we will make the ultraviolet behavior of the individual graphics worse in each consecutive perturbative order, implicating an apparent non-renormalizability.

On the other hand, we can expand an equation analogous to (27) for the regularized fermion propagator $\tilde{G}_{V}^{\Lambda}(p)$ in powers of the function $f_{V}^{\Lambda}$, and show that it is a series in $\hbar^{n}$, as in the usual loop expansion. Writing $\hbar$ explicitly, we get

$$
\begin{aligned}
\frac{1}{\hbar} \tilde{G}_{V}^{\Lambda}(p)=\frac{i}{\not p}+ & e^{2} \hbar \int \frac{d k}{(2 \pi)^{2}} f_{V}^{\Lambda}(k) \frac{1}{\not p} \not k \frac{1}{\not p-\not k}+ \\
& -i e^{4} \hbar^{2} \int \frac{d k}{(2 \pi)^{2}} \frac{d s}{(2 \pi)^{2}} f_{V}^{\Lambda}(k) f_{V}^{\Lambda}(s) \frac{1}{\not p} \not k \frac{1}{\not p-\not k} \$ \frac{1}{\not p-\not k-\$}+\ldots
\end{aligned}
$$


In fact, we can show that expressions (85) and (48) are equivalent to a loopwise expansion using the exact photon propagator, and in this case a power $\left(f_{V(C)}\right)^{n}$ corresponds to $\hbar^{n}$, or $n$ loops. Let us take the second term in expression (85) and define

$$
\tilde{G}_{1}(p ; \Lambda)=e^{2} \hbar \int \frac{d k}{(2 \pi)^{2}} f_{V}^{\Lambda}(k) \frac{1}{\not p} \not k \frac{1}{\not p-\not k} .
$$

We are going to show that

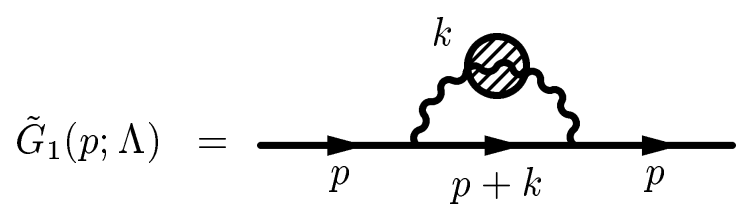

where

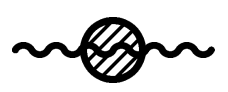

denotes the regularized full photon propagator. The graph in (87) is expressed, in momenta space, as

$$
\tilde{G}_{1}(p ; \Lambda)=e^{2} \int \frac{d k}{(2 \pi)^{2}} \frac{1}{\not p} \gamma^{\mu} \frac{1}{\not p+\not k} \gamma^{\nu}\left(\frac{g_{\mu \nu}}{k^{2}-m^{2}(a)}+f_{V}^{\Lambda}(k) k_{\mu} k_{\nu}\right) \frac{1}{\not p}
$$

The first term, proportional to $g_{\mu \nu}$, is cancelled because, in two dimensions, $\gamma^{\mu} \gamma^{\nu} \gamma_{\mu}=0$. So,

$$
\tilde{G}_{1}(p ; \Lambda)=e^{2} \int \frac{d k}{(2 \pi)^{2}} f_{V}^{\Lambda}(k) \frac{1}{\not p} \not k \frac{1}{p+\not k} \not k \frac{1}{p} .
$$

Using the identity,

$$
\frac{1}{p p} \not k \frac{1}{p+\not k}=\frac{1}{\not p}-\frac{1}{p+\not k}
$$

and the fact that $\not b \phi c=\not b \phi$, we obtain

$$
\tilde{G}_{1}(p ; \Lambda)=e^{2} \int \frac{d k}{(2 \pi)^{2}} f_{V}^{\Lambda}(k)\left(\frac{1}{\not p} \not k \frac{1}{p}-\frac{1}{\not p} \not k \frac{1}{p+\not k}\right) .
$$

The first term inside parenthesis is cancelled because it is the integral of an odd function of $k$. Changing $k$ by $-k$ we arrive where we wanted.

To proceed, let us define

$$
\tilde{G}_{2}(p ; \Lambda)=-i e^{4} \hbar^{2} \int \frac{d k}{(2 \pi)^{2}} \frac{d s}{(2 \pi)^{2}} f_{V}^{\Lambda}(k) f_{V}^{\Lambda}(s) \frac{1}{\not p} \not k \frac{1}{\not p-\not k} \phi \frac{1}{\not p-\not k-\not}
$$


We are going to show that,

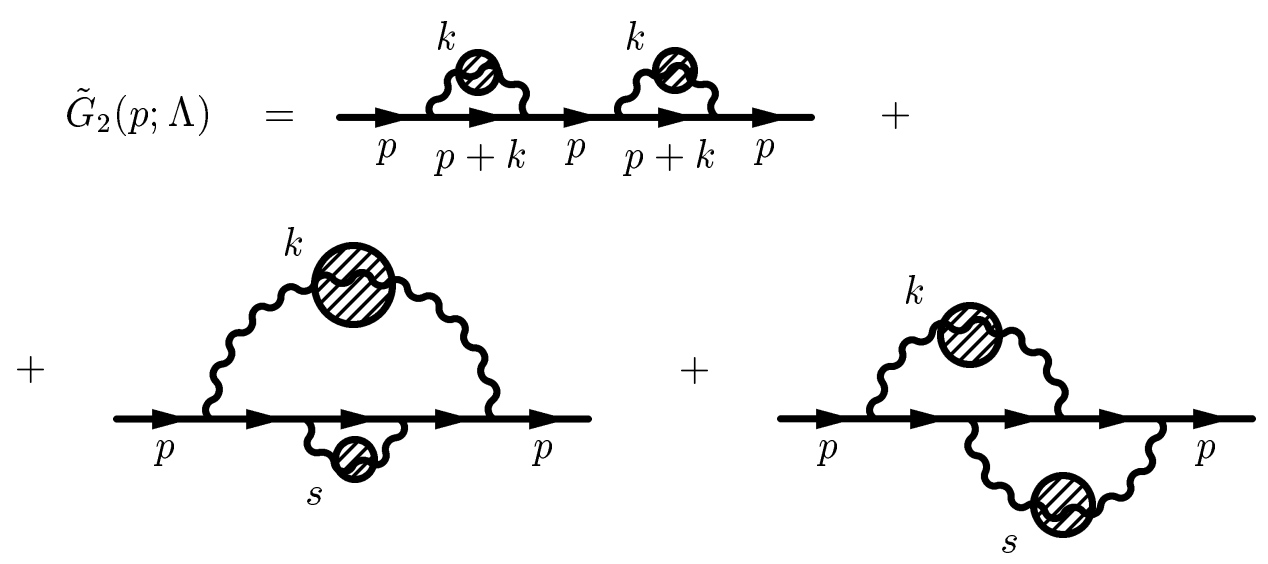

Computing the first graph in (93), we obtain

$$
\begin{gathered}
\tilde{G}_{21}(p ; \Lambda)=\underset{p+k}{\rightarrow p} \underset{p+k}{k} \\
=-i e^{4} \int \frac{d k}{(2 \pi)^{2}} \frac{d s}{(2 \pi)^{2}} f_{V}^{\Lambda}(k) f_{V}^{\Lambda}(s) \frac{1}{\not p} \not k \frac{1}{p+\not k} \not k \frac{1}{p p} \phi \frac{1}{p+\phi} \phi \frac{1}{p} \\
=-i e^{4} \int \frac{d k}{(2 \pi)^{2}} \frac{d s}{(2 \pi)^{2}} f_{V}^{\Lambda}(k) f_{V}^{\Lambda}(s)\left(\frac{1}{\not p}-\frac{1}{\not p+\not k}-\frac{1}{\not p+\not k} \not k \frac{1}{\not p+\not}\right),
\end{gathered}
$$

The second graph is,

$$
\begin{aligned}
& \tilde{G}_{22}(p ; \Lambda)=e^{4} \int \frac{d k}{(2 \pi)^{2}} \frac{d s}{(2 \pi)^{2}} f_{V}^{\Lambda}(k) f_{V}^{\Lambda}(s) \frac{1}{\not p} \frac{1}{p+\not k} \phi \frac{1}{p+\not k+\not k} \not \frac{1}{p+\not k} \not k \frac{1}{\not p} \\
& =-i e^{4} \int \frac{d k}{(2 \pi)^{2}} \frac{d s}{(2 \pi)^{2}} f_{V}^{\Lambda}(k) f_{V}^{\Lambda}(s)\left(\frac{1}{\not p}-\frac{1}{\not p+\not k}+\frac{1}{\not p} \not k \frac{1}{p+\not k+\not k} \not k \frac{1}{p}\right)
\end{aligned}
$$


The third one is,

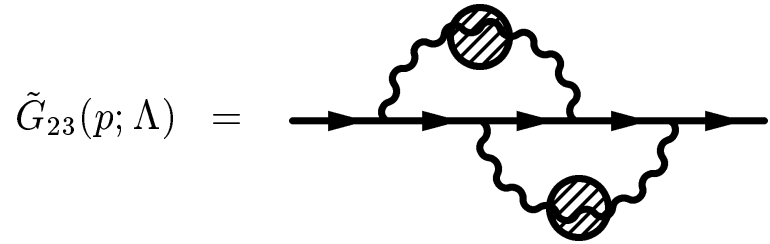

$$
\begin{aligned}
& =-i e^{4} \int \frac{d k}{(2 \pi)^{2}} \frac{d s}{(2 \pi)^{2}} f_{V}^{\Lambda}(k) f_{V}^{\Lambda}(s) \frac{1}{\not p} \not k \frac{1}{\not p+\not k} \$ \frac{1}{\not p+\not k+\not} \not k \frac{1}{\not p+\phi} \$ \frac{1}{\not p} \\
& =-i e^{4} \int \frac{d k}{(2 \pi)^{2}} \frac{d s}{(2 \pi)^{2}} f_{V}^{\Lambda}(k) f_{V}^{\Lambda}(s)\left(-\frac{1}{\not p}+\frac{1}{\not p+\not k}+\right. \\
& \left.-\frac{1}{p} \not k \frac{1}{p+\not k+\not} \not k \frac{1}{p}+\frac{1}{\not p+\not k} \not k \frac{1}{p+\phi}-\frac{1}{\not p} \not k \frac{1}{p+\not k+\phi}\right) \text {. }
\end{aligned}
$$

Summing equations (96), (98) and (100), we obtain

$$
\tilde{G}_{2}(p ; \Lambda)=-i e^{4} \int \frac{d k}{(2 \pi)^{2}} \frac{d s}{(2 \pi)^{2}} f_{V}^{\Lambda}(k) f_{V}^{\Lambda}(s)\left(\frac{1}{\not p}-\frac{1}{p+\not k}-\frac{1}{\not p} \not k \frac{1}{\not p+\not k+\not}\right) .
$$

Finally, identity (90) applied twice gives us what we wanted to proof. The theorem is easily established by finite induction and shown to be valid to all orders. The generalization for the chiral Schwinger model is straightforward.

This expansion of the exact fermion propagator in terms of the exact photon propagator has been found previously in the literature [23, 28, 31]. However, the fundamental role that it plays in the renormalization of two-dimensional gauge theories has not been previously noticed or used. A power counting analysis of expansion (85), with $f^{\Lambda}$ being $f_{V}^{\Lambda}$ or $f_{C}^{\Lambda}$, shows the presence of a regularized UV logarithmic divergence (try to compute the same graphs using $f$ instead of $f^{\Lambda}$ ) in every loop that appears in the fermion propagator. This divergence is similar to the one present in a Proca theory with fermions, due to the bad high momentum behavior of the exact photon propagator. However, it is possible to renormalize the theory in $(1+1)$ dimensions, because the bosonic sector is only quadratic in the field $A_{\mu}$, after fermion integration. As we are going to see, the renormalization of the fermionic sector can be done without problems, once we recognize that we have to use the exact photon propagator, instead of the tree level one.

The exact photon propagator itself does not exhibit the divergence of the tree level one, which is cancelled when we add the terms of the geometric sum that defines it. This shows that, in these anomalous theories, the exact or complete photon propagator has to be considered instead of the tree level one. The tree level of the bosonic sector is thus indefinite. We call this kind of approach a semi-perturbative one. 


\subsection{Renormalization to 1-loop order}

Now, we can calculate the 1PI functions to 1-loop order (in this semi-perturbative sense) and impose renormalization conditions, to determinate the finite part of the renormalization constants.

The regularized 1PI two point fermion function (48) $\tilde{\Gamma}_{V}^{\Lambda}$ to 1-loop order is

$$
\tilde{\Gamma}_{V}^{\Lambda}(p)=\not p\left[1+\frac{\hbar}{2\left(a_{V}-1\right)} \ln \left(1-\frac{\Lambda^{2}}{p^{2}}\right)-\frac{\hbar}{2\left(a_{V}+1\right)} \ln \left(1-\frac{m_{V}^{2}}{p^{2}}\right)\right]
$$

The renormalized $\tilde{\Gamma}_{V}^{R}$ function is given by

$$
\tilde{\Gamma}_{V}^{R}=Z_{\psi}^{V} \tilde{\Gamma}_{V}^{\Lambda}
$$

where $Z_{\psi}^{V}$ is the renormalization constant of the fermion field. The next step, is the imposition of the renormalization conditions that can fix the finite part of the renormalization constants. This can be done by requiring that

$$
\left.\tilde{\Gamma}_{V}^{R}(p)\right|_{p=\mu^{k}}=\mu .
$$

Using it, we get $Z_{\psi}^{V}$ to 1-loop order,

$$
Z_{\psi}^{V}=1-\frac{\hbar}{2\left(a_{V}-1\right)} \ln \left(1-\frac{\Lambda^{2}}{\mu^{2}}\right)+\frac{\hbar}{2\left(a_{V}+1\right)} \ln \left(1-\frac{m_{V}^{2}}{\mu^{2}}\right) .
$$

To this order, we get $\tilde{\Gamma}_{V}^{R}$ as

$$
\tilde{\Gamma}_{V}^{R}(p)=\not p\left[1+\frac{\hbar}{a_{V}^{2}-1} \ln \left(\frac{\mu^{2}}{p^{2}}\right)-\frac{\hbar}{2\left(a_{V}+1\right)} \ln \left(\frac{1-p^{2} / m_{V}^{2}}{1-\mu^{2} / m_{V}^{2}}\right)\right] .
$$

The computation up to one loop of the 1PI 2-point function for the chiral case will also be done. Defining the regularized right-handed 1PI 2-point function as $\tilde{\Gamma}_{C}^{\Lambda_{+}}$, we obtain

$$
\tilde{\Gamma}_{C}^{\Lambda^{+}}(p)=\not p P_{+}\left[1+i \hbar e^{2} \int \frac{d k}{(2 \pi)^{2}} f_{C}^{\Lambda}(k) \not k \frac{1}{p-\not k}+\mathcal{O}\left(\hbar^{2}\right)\right] .
$$

The function $f_{C}^{\Lambda}$ can be written as

$$
\begin{aligned}
f_{C}^{\Lambda}(k)=- & \frac{\left[\omega_{\Lambda}-a_{C} \Lambda^{2} /\left(a_{C}-1\right)\right]^{2}}{\omega_{\Lambda}\left(\omega_{\Lambda}-\omega_{m}\right)} \frac{1}{\left(k^{2}-\Lambda^{2}\right)\left(k^{2}-\omega_{\Lambda}\right)}+ \\
& +\frac{\left[\omega_{m}-a_{C} \Lambda^{2} /\left(a_{C}-1\right)\right]^{2}}{\omega_{m}\left(\omega_{\Lambda}-\omega_{m}\right)} \frac{1}{\left(k^{2}-\Lambda^{2}\right)\left(k^{2}-\omega_{m}\right)} .
\end{aligned}
$$


We compute the integral in (107) to obtain

$$
\tilde{\Gamma}_{C}^{\Lambda}(p)=\not p P_{+}\left[1-\frac{\hbar}{a-1} \ln \left(\frac{\Lambda^{2}}{m_{C}^{2}}\right)+\frac{\hbar}{a-1} \ln \left(1-\frac{p^{2}}{m_{C}^{2}}\right)\right]
$$

Now, we renormalize this $1 \mathrm{PI}$ function $\tilde{\Gamma}_{C}^{R+}=Z_{\psi}^{C} \tilde{\Gamma}_{C}^{\Lambda_{+}}$and use the renormalization condition (104) to get the one loop wave function renormalization constant

$$
Z_{\psi}^{C}=1+\frac{\hbar}{a-1} \ln \left(\frac{\Lambda^{2}}{m_{C}^{2}}\right)-\frac{\hbar}{a-1} \ln \left(1-\frac{\mu^{2}}{m_{C}^{2}}\right) .
$$

Then the renormalized 1PI 2-point function to one loop order is

$$
\tilde{\Gamma}_{C}^{R}(p)=\not p P_{+}\left[1+\frac{\hbar}{a_{C}-1} \ln \left(\frac{1-p^{2} / m_{C}^{2}}{1-\mu^{2} / m_{C}^{2}}\right)\right] .
$$

\section{Conclusions}

We have seen, through the examples of the vector and chiral Schwinger models, how to renormalize an anomalous gauge theory, at least in two dimensions. The main feature is that the theory is renormalizable, in the usual sense, if the complete photon propagator can be computed. This could be a good starting point to attack the same question in four dimensions, if we could estimate or take into account the main characteristics of the exact photon propagator.

In the regularized version of the theory, the dependence on $a$ is completely contained in the cut-off dependent "gauge fixing" parameter. If we were dealing with a "normal" gauge theory, this would suggest that the complete theory (that one where the gauge field is also quantized) is independent of $a$, thus allowing one to choose whether one keeps or not explicit gauge invariance in any intermediate steps of the quantization. However, we must be careful in analysing this, because of the apparently non-gauge invariant nature of the quantum theory. It is necessary to remember here that we are not fixing the gauge (we do not have the right to do that, it is an anomalous theory), but regularizing divergences of non-perturbative nature.

Finally, we call attention to the fact that, from the point of view of a semiperturbative renormalization, one does not distinguish between a truly anomalous gauge theory and a non-anomalous. It is only when the gauge field is fully quantized that one can see this clearly, what is not usually done. Most works limit themselves to integrating over the fermion fields and, forgetting fermionic sources, manipulating a quadratic bosonized theory. For many applications, this is not wrong. But, as we have shown, many interesting aspects of the theory are revealed only with the complete quantization.

We are currently investigating the physical consequences of these renormalized versions of the theories. We can say, preliminarily, that the parameter $a$ is 
apparently controlling the screening and confinement properties. Progress in this direction will be reported elsewhere.

\section{Acknowledgment}

This work was supported by Conselho Nacional de Desenvolvimento Científico e Tecnológico, CNPq, Brazil.

\section{References}

[1] S. Weinberg, The Quantum Theory of Fields, vols. 1 and 2, Cambridge University Press, New York, 1996.

[2] T.-P. Cheng and L.-F. Li, Gauge Theory of Elementary Particle Physics, Clarendon Press, London, 1984.

[3] M. B. Green, J. H. Schwarz and E. Witten, Superstring Theory, vols. 1 and 2, Cambridge University Press, New York, 1987.

[4] R. Jackiw and R. Rajaraman, Phys. Rev. Lett., 54, (1985) 1219.

[5] J. Schwinger, Phys. Rev., 128, (1962), 2425.

[6] R. Jackiw, Topological Investigations of Quantized Gauge Theories, in Relativity, Groups and Topology II (Les Houches 1983), eds. B.S. DeWitt and R. Stora, North Holland, Amsterdam, 1984.

[7] J. Lowenstein and J. Swieca, Ann. Phys., 68, (1971) 172.

[8] S. Coleman, R. Jackiw and L. Susskind, Ann. Phys., 93, (1975) 267.

[9] N. K. Nielsen and B. Schroer, Nucl. Phys., B120 (1977) 62.

[10] K. D. Rothe and J. A. Swieca, Ann. Phys., 117, (1979) 382.

[11] R. Roskies and F. Schaposnik, Phys. Rev., D23, (1981) 558.

[12] M. Hortacsu, K. D. Rothe and B. Schroer, Phys. Rev., D20, (1979) 3203.

[13] C. Jayewardena, Helv. Phys. Acta 65 (1988) 636.

[14] C. Adam, Z. Phys C63 (1994) 169.

[15] N. S. Manton, Ann. Phys., 159, (1985) 220.

[16] R. Jackiw and K. Johnson, Phys. Rev. 182, (1969), 1459. 
[17] P. Mitra and A. Rahaman, Ann. Phys. 249 (1996), 34.

[18] L. D. Faddeev and S. L. Shatashvili, Phys. Lett. B167, (1986), 225.

[19] O. Babelon, F. Schaposnik and C. M. Viallet, Phys. Lett. B177, (1986), 385.

[20] K. Harada and I. Tsutsui, Phys. Lett. B183, (1987), 311.

[21] S. A. Frolov and A. A. Slavnov, Phys. Lett. B269, (1991), 377.

[22] E. Abdalla, M. C. B. Abdalla and K. D. Rothe, Non-Perturbative Methods in 2 Dimensional Quantum Field Theory, World Scientific, Singapore, 1991.

[23] Z. Jian-Ge, D. Qing-Hai and L. Yao-Yang, Phys. Rev. D43 (1991) 613.

[24] H. O. Girotti, H. J. Rothe and K. D. Rothe, Phys. Rev. D34, (1986), 592.

[25] D. Boyanovsky, Nucl. Phys., B294, (1987), 223.

[26] Rodolfo Casana Sifuentes, Renormalização e Ambigüidades na $Q E D_{2}$, Ms.C Thesis, 02/97, CBPF.

[27] Rodolfo Casana S. and Sebastião A. Dias, work in preparation.

[28] C. Adam, Czech. J. Phys. 48 (1998) 9; hep-ph 9601228.

[29] T. Radozycki and J. M. Namyslowski, Phys. Rev. D59, (1999), 065010.

[30] J. Zinn-Justin, Quantum Field Theory and Critical Phenomena, 2 nd edition, Oxford Science Pub., 1993.

[31] T. Radozycki, Eur. Phys. Journ., C6, (1999), 549. 